\title{
Disulfiram Suppresses Invasive Ability of Osteosarcoma Cells Via the Inhibition of MMP-2 and MMP-9 Expression
}

\author{
Hyun-Ji Cho ${ }^{1,2}$, Tae-Sung Lee ${ }^{1,2}$, Jae-Bok Park ${ }^{2}$, Kwan-Kyu Park ${ }^{1,2}$, Jung-Yoon Choe ${ }^{1,2}$, Doo-Il Sin ${ }^{2}$, Yoon-Yub Park ${ }^{1,2}$, \\ Yong-Suk Moon ${ }^{1,2}$, Kwang-Gill Lee ${ }^{3}$, Joo-Hong Yeo ${ }^{3}$, Sang-Mi Han ${ }^{3}$, Young-Su Cho ${ }^{4}$, Myeong-Rak Choi ${ }^{5}$, \\ Nam-Gyu Park ${ }^{6}$, Yun-Sik Lee ${ }^{7}$ and Young-Chae Chang ${ }^{1,2, *}$ \\ ${ }^{1}$ Research Institute of Biomedical Engineering, and \\ ${ }^{2}$ Department of Medicine, Catholic University of Daegu School of Medicine, Daegu 705-718, Korea \\ ${ }^{3}$ Department of Agricultural Biology, National Institute of Agricultural Science and Technology, Suwon 441-100, Korea \\ ${ }^{4}$ Division of Biotechnology, Faculty of Natural Resources and Life Science, Dong-A University, Busan 604-714, Korea \\ ${ }^{5}$ Department of Biotechnology, Chonnam National University, Yeosu, 550-749, Korea \\ ${ }^{6}$ Department of Biotechnology and Bioengineering, Pukyong National University, Busan 608-737, Korea \\ ${ }^{7}$ Department of Medical Zoology, College of Medicine, Kyung Hee University, Seoul 130-701, Korea
}

Received 9 May 2007, Accepted 26 October 2007

\begin{abstract}
Cancer cells, characterized by local invasion and distant metastasis, are very much dependant on the extracellular matrix. The expression of matrix metalloproteinases (MMPs) has been implicated in the invasion and metastasis of cancer cells. In this study, we reported the effects of disulfiram, a clinically used anti-alcoholism drug, on tumor invasion suppression, as well as its effects on the activity of MMP-2 and MMP-9 in human osteosarcoma cells (U2OS). Disulfiram has been used for alcohol aversion therapy. However, recent reports have shown that disulfiram may have potential in the treatment of human cancers. Herewith, we showed that the anti-tumor effects of disulfiram, in an invasion assay using U2OS cells and that disulfiram has a type IV collagenase inhibitory activity that inhibits expression of genes and proteins responsible for both cell and non-cell mediated invasion on pathways. In conclusion, disulfiram inhibited expression of MMP-2 and MMP-9 and it regulated the invasion of human osteosarcoma cells. These observations raise the possibility of disulfiram being used clinical for the inhibition of cancer invasion.
\end{abstract}

Keywords: Disulfiram, Invasion, MMP-2, MMP-9, U2OS

*To whom correspondence should be addressed. Tel: 82-53-650-4848; Fax: 82-53-650-4834

E-mail: ycchang@cu.ac.kr

\section{Introduction}

Osteosarcoma is the most common primary malignant tumor of the bone and is known to metastasize. Changes in the cellextracellular matrix (ECM) interactions, dissociation of intercellular adhesion complexes and degradation of ECM are needed for metastasis of osteosarcoma to occur (Yoon et al., 2001; Stewart et al., 2004). Degradation and dissociation of the ECM is a special feature of tumor invasion, metastasis and angiogenesis in osteosarcoma.

The degradation of ECM has been linked to MMPs. MMPs are known to be closely associated with tumor growth and metastasis as well (Matsunaga et al., 2004). MMPs are a family of zinc endopeptidases consisting of at least 20 different members (Soini et al., 2001; Yasuhito et al., 2002) and they play an important role in various cellular metabolic processes. They induce a variety of biological effects including growth, morphogenesis, apoptosis, tissue destruction, and cancer formation (Lee et al., 2005; Soreide et al., 2006). Among the human MMPs reported previously, two gelatinases, gelatinase A (72-kDa type VI collagenase, MMP-2) and gelatinase B (92-kDa type VI collagenase, MMP-9) can degrade type IV collagen and fibronectin, and are closely related to the invasion and metastasis of tumor cells (Liotta et al., 1991; Masahiro et al., 2002; Tanimura et al., 2005).

MMP-2 and MMP-9 expression are known to be regulated by transcription factors, AP-1, NF- $\kappa \mathrm{B}$ and $\mathrm{Sp}-1$ (Woo et al., 2004). It was recently found that AP-1 regulates downstream targets such as the matrix metalloproteinase-9 promoter in human renal carcinoma cells (Hong et al., 2005; Cho et al., 2007). Although MMPs are associated with invasion and 
metastasis, the mechanisms of MMP-2 and MMP-9 expression in tumor cells remain largely unknown.

Disulfiram (DSF) has been used for over half a century for alcohol aversion therapy (Shen et al., 2000). Disulfiram is a chelator of heavy metals and an inhibitor of aldehyde dehydrogenase (Marikovsky et al., 2003). Chelation is the process of reversible binding of a ligand to a meal ion, forming a metal-chelator complex. Chelators are used in chemical analysis and are also employed to safely bind poisonous metal agents such as mercury, arsenic or lead, to stabilize them and allow them to be excreted without further interaction within the body. Commonly used synthetic chelators include iron-chelating agents such as EDTA (Li et al., 2007), D-penicillamine (DPA) and 2,3 dimercaptosuccinic acid (DMSA) (Dhawan et al., 1989). Recent reports indicate that disulfiram and other dithiocarbamates may have a significant potential in the treatment of human cancers (Chen et al., 2006). Disulfiram has also been shown to inhibit the activation of the transcription factor/cyclic-AMP-responsive element binding protein, which is implicated in the growth and progression of melanomas (Brar et al., 2004). These preceding results reveal that disulfiram may have an important role as an adjuvant in the chemotherapy of human cancers. In this paper, we report the effects of disulfiram on type IV collagenase, MMP-2 and MMP-9 in human osteosarcoma cells and its suppression-related mechanism. These results provide evidence that the invasion phenotype of tumorsis correlated with MMP-2 and MMP-9 activity, suggesting that inhibition of MMP-2 and MMP-9 activity via the chelating agent disulfiram may serve as a way to inhibit human osteosarcoma cell invasion.

\section{Materials and methods}

Cell culture and conditioned medium. Cell lines used in the study were maintained in humidity in a $5 \% \mathrm{CO}_{2}$ incubator. U2OS (human osteosarcoma), Caki-1 (renal carcinoma), Caski (cervical carcinoma cell), MDA-MB-231 (human breast cancer) cells were obtained from the American Type Culture Collection. Cells were cultured in DMEM-High glucose (Dulbecco's Modified Eagle's medium) (GibcoBRL) medium containing 10\% fetal bovine serum (Hyclone Laboratories) and 1\% ampicillin (Gibco). After a $24 \mathrm{~h}$ attachment, the medium was replaced with serum-free medium and various dose of disulfiram (SIGMA) and PMA (phorbol 12-myristate 13acetat) (SIGMA) were added.

Soft agar colony formation assay. Effects of disulfiram on the soft agar colony formation of U2OS cells were investigated. Single-cell suspensions of U2OS cells were treated with or without different concentrations of disulfiram, and then mixed with agarose in a final concentration of $0.35 \%$. Aliquots of $1.5 \mathrm{ml}$ containing $10^{4}$ cells and $10 \%$ FBS were plated in triplicate in $60 \mathrm{~mm}$ culture dishes over a base layer of $0.7 \%$ agarose and allowed to solidify. Following 14 days of incubation, the colonies were stained with $0.005 \%$ crystal violet and those which were $>60 \mu \mathrm{m}$ were counted using a dissecting microscope.
Cell viability assay. Reduction of 3-[4,5-dimethlyhiazol-2-yl]-2,5diphenyltetrazolium bromide (MTT) (Roche Applied Science) assays were performed as described in the supplier's protocol to evaluate the cytotoxicity of disulfiram.

Cell invasion assay. For invasion assay, $5 \times 10^{4}$ cells were seeded in the upper well of each transwell chamber. Before invasion assay, the upper parts of the transwell (Corning Costar) were coated with $30 \mu \mathrm{l}$ of a $1: 2$ mixture of matrigel : phosphate-buffered saline. Cells were plated on the matrigel-coated transwells in the presence of various concentrations of disulfiram. Culture media was placed in the lower compartment of the cemotaxis chamber as a source of chemoattractants. The inserts were incubated for $24 \mathrm{~h}$ at $37^{\circ} \mathrm{C}$. Cells that had invaded the lower surface of the membrane were fixed with methanol and stained with hematoxylin and eosin. Using light microscopy, random fields were selected and cells in each field were counted under high power $(\times 200)$.

Zymographic assay for metalloproteinase. Zymography was used to determine the effect of disulfiram on PMA-induced MMP-9 activity after the cells were treated with disulfiram in the presence of $75 \mathrm{nM}$ PMA. Cell were suspended in medium containing $10 \%$ fetal bovine serum and plated at $3 \times 10^{5}$ cells $/ 60 \mathrm{~mm}$ dish. The dishes were incubated until the cultures were $80 \%$ confluent. The medium was then changed to fresh serum-free medium with or without disulfiram. Supernatant was collected after incubation for $24 \mathrm{~h}$. The cellected cell culture medium was mixed with $4 \times$ SDS sample buffer (without $\beta$-mercaptoethanol) for electrophoresis. Sample was loaded onto the SDS-PAGE in $10 \%$ polyacrylamide gel containing $0.1 \%$ gelatin and electrophoresed. After electrophoresis, the gels were washed thrice in $2.5 \%$ triton $\mathrm{X}-100$ for $10 \mathrm{~min}$ at room temperature, then incubated for $24 \mathrm{~h}$ at $37^{\circ} \mathrm{C}$ in incubation buffer containing $5 \mathrm{mM} \mathrm{CaCl}_{2}$ and $1 \mu \mathrm{M} \mathrm{ZnCl}_{2}$. Gels were stained with $0.25 \%$ Coomassie Brilliant Blue R250 for $1 \mathrm{~h}$ and then de-stained for $30 \mathrm{~min} \sim 1 \mathrm{~h}$ at room temperature. Proteolytic activity was visible in the form of clear bands against the blue background of the stained gelatin.

Western Bolt Analysis. Cellular lysates were prepared by suspending $3 \times 10^{5}$ cells $/ 60 \mathrm{~mm}$ plates in lysis buffer. The cells were disrupted and extracted at $4^{\circ} \mathrm{C}$ for $1 \mathrm{~h}$. After $1 \mathrm{~h}$, they were centrifuged and the supernatant containing proteins were collected into new e-tube. Each lane was loaded with samples on 10\% SDS-PAGE gel, separated and electro-transferred to immobilon-p membranes (Millipore Co.). Detection of specific proteins was carried out by enhanced chemiluminescence following the manufacture's instructions (Amersham Biosciences). MMP-9 and MMP-2 rabbit polyclonal antibodies were purchased from Chemicon (Chemicon International).

Isolation of RNA and RT-PCR. Total cellular RNA was isolated using a RNA ZolBee (Life Technology). For reverse transcription reaction, cDNA was synthesized from total RNA using moloney murin leukemia virus reverse transcriptase (Promega). PCR primers sequences used were as follows: MMP-2 sense 5'-GGCCCTGTCA CTCCTGAGAT-3' and antisense 5'-GGCATCCAGGTTATCGGG GA-3'; MMP-9 sense 5'-CGGAGCACGGAGACGGGTAT-3' and antisense 5'-TGAAGGGGAAGACGCACAGC-3'. PCR products were resolved electrophoretically on a $1.0 \%(\mathrm{w} / \mathrm{v})$ agarose gel, and 
DNA bands were visualized by staining with ethidium bromide. The expression of measured genes in each sample was normalized to $\beta$-actin expression. All samples were analyzed in triplicate.

Luciferase reporter gene assay. MMP-2 wild type (pGL3-MMP2WT-luc) luciferase promoter constructs and MMP-9 wild type (pGL3-MMP-9WT-luc) luciferase promoter constructs were used in transient transfection assays. U2OS cells were plated at a density of $3 \times 10^{5}$ cells $/ 60 \mathrm{~mm}$ dish and grown to $60 \%$ confluence in $2 \mathrm{ml}$ of growth medium. The cells were transiently transfected with $5 \mu \mathrm{g}$ of various plasmid constructs for $6 \mathrm{~h}$ using lipofectamine reagent (Invitrogen) according to the manufacturer's instructions. After transfection, the cells were cultured in serum-free medium with $20 \mu \mathrm{M}$ disulfiram for $24 \mathrm{~h}$. The cells were then washed twice with cold PBS and lysed with reporter lysis buffer (Promega). After centrifugation, cell extract and luciferase assay reagent were mixed at room temperature and placed in a luminometer (Luminometer, Microlumat Plus, Berthold Technologies, Dortmund, Germany) to detect the light produced.

Statistical analysis. All values were expressed as mean \pm S.E. Each value is the mean of at least three repetitive experiments in each group. T-test was used to compare the effect of the compounds and their difference from the vehicle-treated control was analyzed for significance.

\section{Results}

Disulfiram suppresses MMP-2 and MMP-9 activity. The gelatin zymography assay showed that the secretion of MMP9 in the conditioned medium of U2OS, Caski and Caki-1 cells were dramatically induced by PMA, and activity of MMP-2 and MMP-9 was decreased by DSF (Fig. 1). As shown in Fig. 1 , treatment of U2OS with disulfiram, at doses above $20 \mu \mathrm{M}$, strongly suppressed PMA-induced MMP-9 and MMP-2 activity compared to caki-1 and caski cells in a dosedependent manner.

These results indicate that disulfiram inhibits MMP-2 and MMP-9 activity in U2OS more than the other cell lines tested.

Effect of disulfiram on growth and proliferation of human osteosarcoma cells. It has been reported that the ability of cells to form colonies in a semi-solid medium is generally considered a marker of anchorage independence and is positively correlated with metastatic potential. The soft agar colony formation assay showed that disulfiram significantly reduced the soft agar colony forming capacity of osteosarcoma cells. The colonies that appeared were scanty and were small in size (Fig. 2A-b and B). The results also revealed a dosedependent effect and at $20 \mu \mathrm{M}$, disulfiram inhibited colony formation by $95 \%$ (Fig. 2A-b and B). Disulfiram cytotoxicity as a cause of the above results was eliminated when cell viability testing on U2OS cells treated with $10 \mu \mathrm{M}$ of disulfiram for $24 \mathrm{~h}$ showed that the viability decreased by only 10 to $20 \%$ (Fig. 2C).

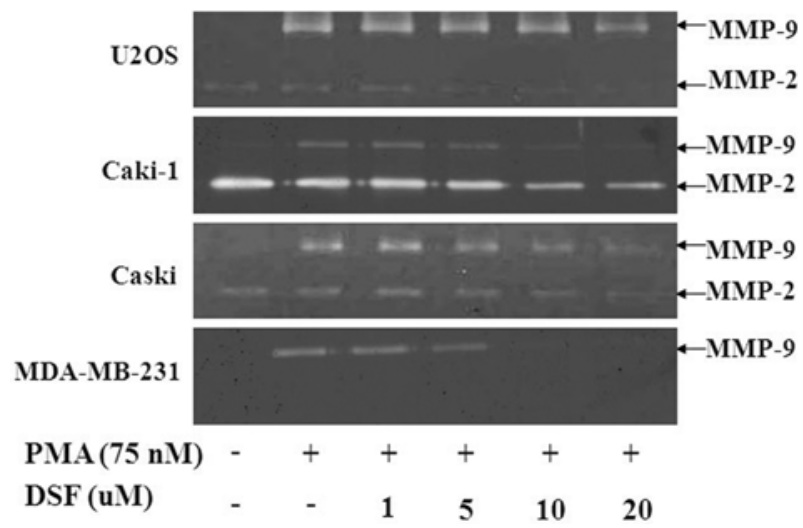

Fig. 1. Effects of disulfiram in various cell lines. Various cell lines were treated with different concentrations of disulfiram in the presence of PMA $(75 \mathrm{nM})$. Conditioned medium was collected from cultures after $24 \mathrm{~h}$ and analyzed by gelatin zymography. The data represents the mean \pm S.E. of at least three independent experiments. Results were deemed statistically significant using Student's $t$-test.

Inhibitory effect of disulfiram on the invasion of human osteosarcoma cells in an in vitro assay. Transwell assay was performed to further evaluate the anti-invasion activity of disulfiram. As shown in Fig. 2D, disulfiram inhibited PMAdependent invasion in a dose-dependent manner, with in the $10 \sim 20 \mu \mathrm{M}$ disulfiram treated cells reaching the levels similar to the control cells. Our data suggested that disulfiram counteracts the effects of PMA that induces the invasive activity in human osteosarcoma cells.

Effects of disulfiram on the MMP-2 and MMP-9 activities of human osteosarcoma cells. U2OS cells, which release MMP-2 and MMP-9 when cultured in serum-free medium, were treated with various concentrations of PMA for $24 \mathrm{~h}$. The level of MMP-2 expression was not significantly altered by PMA $(75 \mathrm{nM})$, but the expression of MMP-9 was induced, as evidenced by gelatin zymography (Fig. 3 and data not shown). From 1 to $20 \mu \mathrm{M}$, disulfiram caused a dose-dependent decreases in PMA-induced MMP-9 activity as well as the MMP-2 activity (Fig. 3). These findings demonstrated that disulfiram inhibits type VI collagenase activity, especially for MMP-2 and MMP-9. Therefore, this inhibition maybe mediated largely through direct drug-protein interaction rather than by the cell-mediated pathway.

Effects of disulfiram on the MMP-2 and MMP-9 protein expression of human osteosarcoma cells. The levels of MMP-2 and MMP-9 proteins in the cells were analyzed in order to explore whether the decreased enzyme activity was due to a diminished amount of MMP-2 and MMP-9 proteins. Our data revealed that the amount of MMP-2 and MMP-9 proteins were decreased in the cells treated with disulfiram (Fig. 4A and B). Similar to the zymographic data, it revealed that the amount of MMP-9 protein decreased in a dose- 
(A)

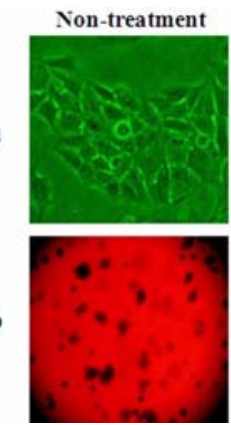

(C)

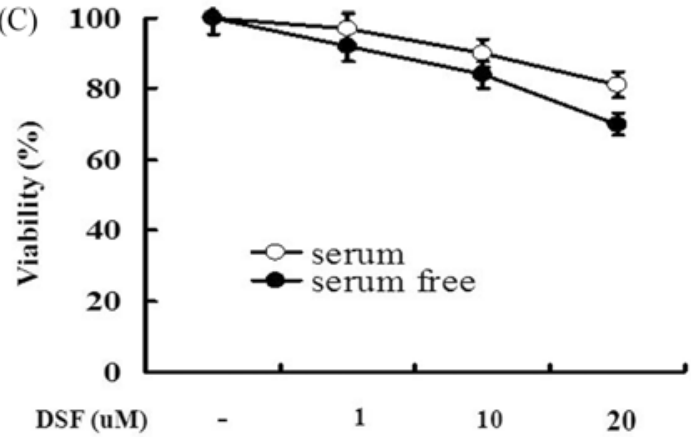

(B)
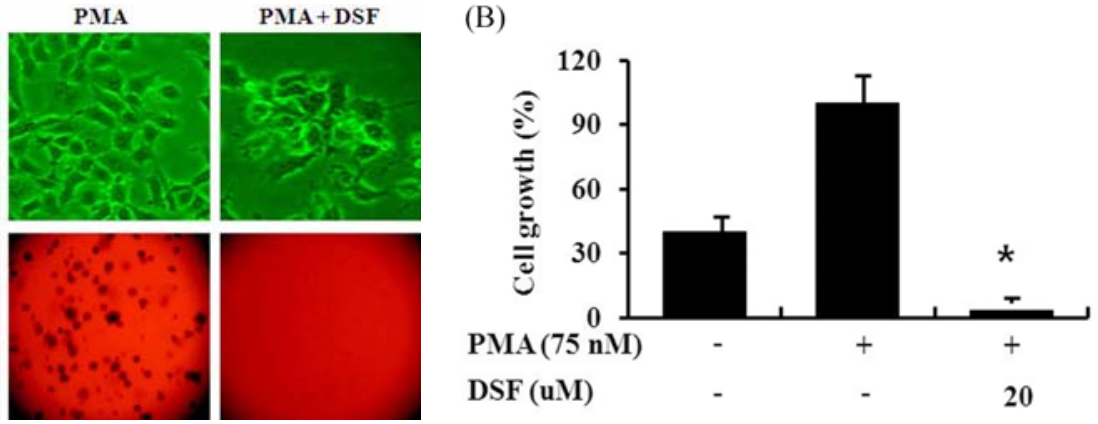

(D)

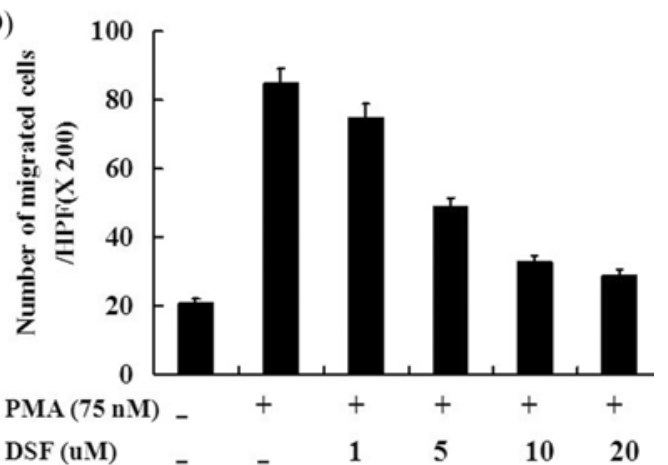

Fig. 2. Effects of disulfiram in U2OS cells. Effects of disulfiram on morphology (A-a), soft agar colony formation assay (A-b) and quantification by colony formation (B) in U2OS cells. (A-a) U2OS cells were treated with PMA (75 nM) and disulfiram (20 uM). Cell is detected under light microscopy. (A-b) U2OS cells were treated with disulfiram in $0.35 \%$ agarose containing $10 \%$ FBS over $0.7 \%$ agarose containing 10\% FBS. Cell colonies were counted after 15-days under light microscopy. C. Cytotoxicity of U2OS cells treated with different concentrations of disulfiram. U2OS cell viability was tested by MTT assay after $24 \mathrm{~h}$. D. Effects of disulfiram in vitro invasion of U2OS cells. For invasion assays, the upper parts of trans-wells were coated with Matrigel, and U2OS cells with various concentrations of disulfiram and $75 \mathrm{nM}$ of PMA were added. After $24 \mathrm{~h}$, cells on the bottom side of the filter were fixed, stained, and counted. Data represent the mean \pm S.E. of at least three independent experiments. Results were deemed statistically significant using Student's $t$-test.

dependant manner as compared to that of MMP-2 protein. The reduced MMP-9 activity was also due to the smaller quantity of proteins produced. Our results suggest that disulfiram inhibits the protein expression and activity of MMP-2 and MMP-9.

Suppression of MMP-2 and MMP-9 expression by disulfiram. RT-PCR and reporter gene analysis were done to determine whether the inhibition of MMP-2 and MMP-9 expression by disulfiram was due to a decreased level of transcription. The treatment of U2OS cells with disulfiram showed a decrease in the levels of PMA-induced MMP-9 and MMP-2 mRNA, whereas TIMP (-1 and TIMP-2 remained largely unchanged (Fig. 4C and D). To test which of these transcription factors may regulate the MMP-2 and MMP-9 genes in U2OS cells, cells were transiently transfected with reporter genes that included the wild type (WT) MMP-2 promoter and WT MMP-9 promoter. Luciferase activity in the cells with the WT MMP-2 and WT MMP-9 construct was significantly reduced when treated with disulfiram (Fig. 5). These results indicate that disulfiram inhibits the expression of MMP-2 and MMP-9 through regulation of transcription levels.
Therefore, the inhibition of MMP-2 and MMP-9 are regulated through both non-cell-mediated and cell-mediated pathways.

\section{Discussion}

The metastatic spread of cancer causes $90 \%$ of human cancer related deaths, and as a result, remains one of the greatest barrier to curing cancer (Christofori, 2006). Several proteins are altered in cancer cells that promote their invasive or metastatic capabilities. Among these proteins, tumor-associated matrix metalloproteinases (MMPs) are important components in the metastatic process through their capacity to degrade extracellular matrix proteins. Among the previously reported human MMPs, MMP-2 and MMP-9 are considered to be particularly good targets for anticancer drugs because both enzymes degrade gelatins, which are major components of the basement membrane. The expression of both these enzymes correlated with an aggressive, advanced invasive or metastatic tumor phenotype (Sato and Seiki, 1993; Hong et al., 2005).

Disulfiram is an inhibitor of aldehyde dehydrogenase and is 
(A)

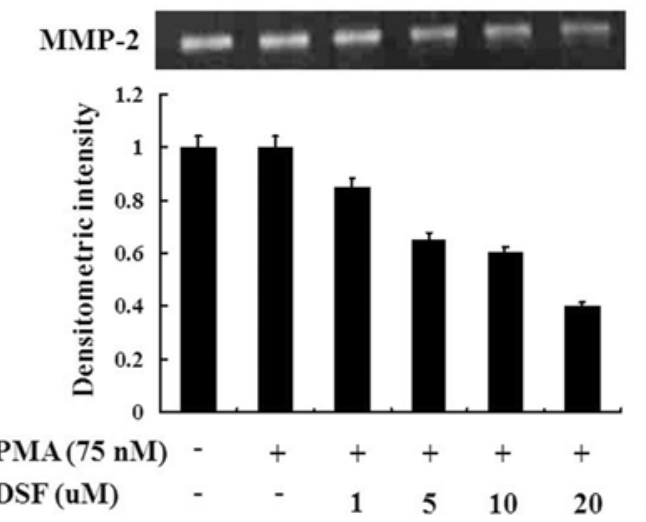

(B)

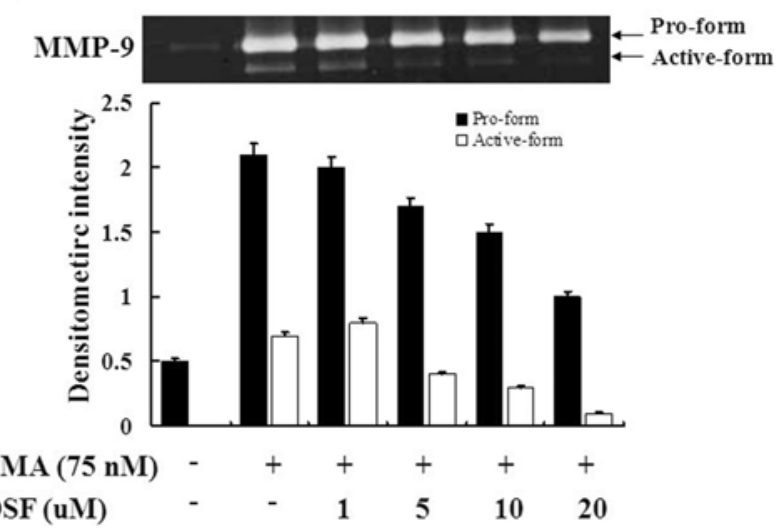

Fig. 3. Effect of disulfiram on MMP-2 activity (A) and PMA-induced MMP-9 activity (B). U2OS cells were treated with various concentrations of disulfiram in the presence of PMA $(75 \mathrm{nM})$. Conditioned medium was collected from cultures after $24 \mathrm{~h}$ and analyzed by gelatin zymography. Data represents the mean \pm S.E. of at least three independent experiments. Results were deemed statistically significant using Student's $t$-test.

(A)

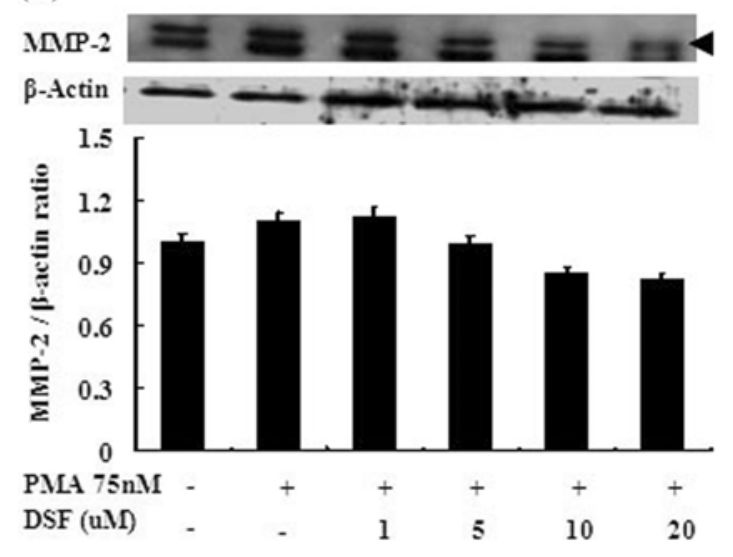

(C)

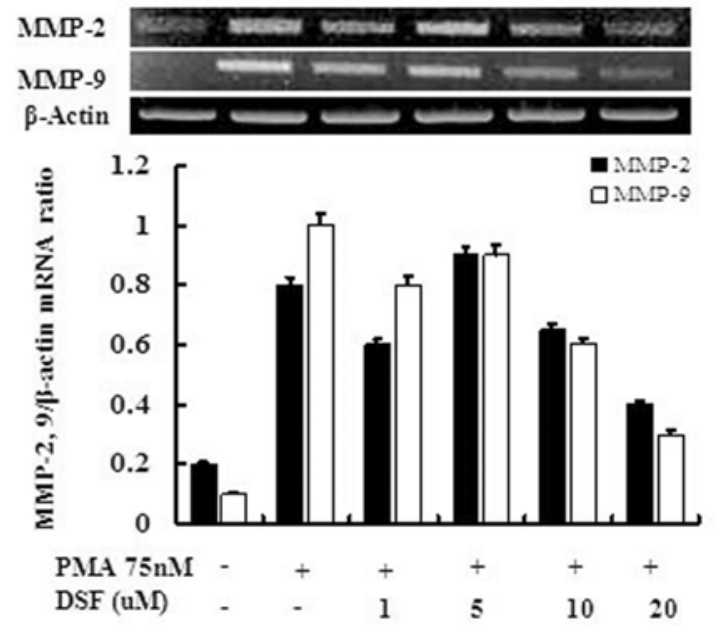

(B)

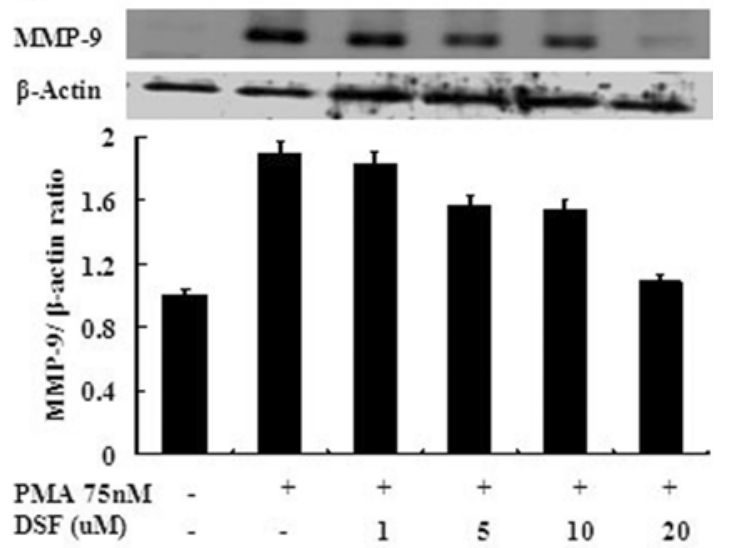

(D)

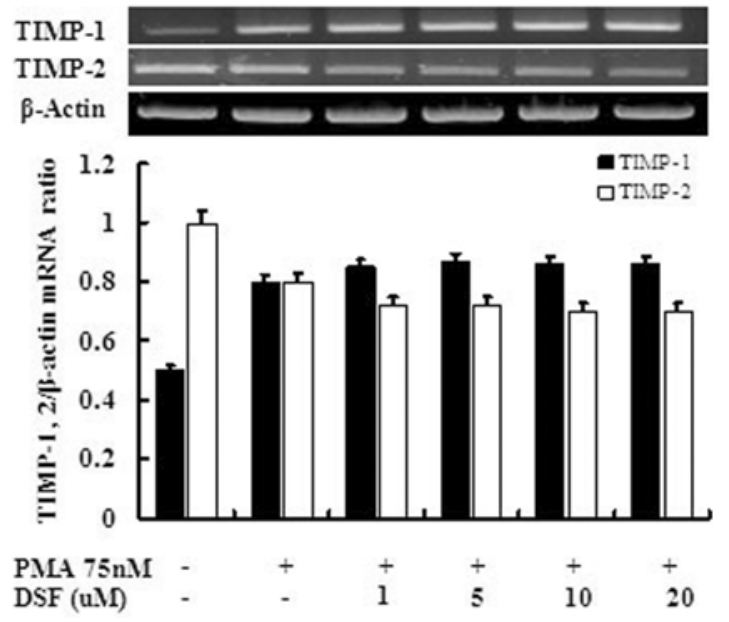

Fig. 4. Effect of disulfiram on MMP-2 and MMP-9 expression and mRNA expression of MMP-2 and MMP-9, TIMP-1 and TIMP-2. A and $\mathrm{B}$, expression of MMP-2 and MMP-9 treated with vehicle and disulfiram in the presence of PMA for $24 \mathrm{~h}$ was evaluated by western blot analysis using anti-MMP-2 and anti-MMP-9. C and D, using RT-PCR, the levels of MMP-2, MMP-9, TIMP-1 and TIMP-2 mRNA from cells treated as indicated were determined. Data represent the mean \pm S.E. of at least three independent experiments. Results were statistically significant using Student's $t$-test. 

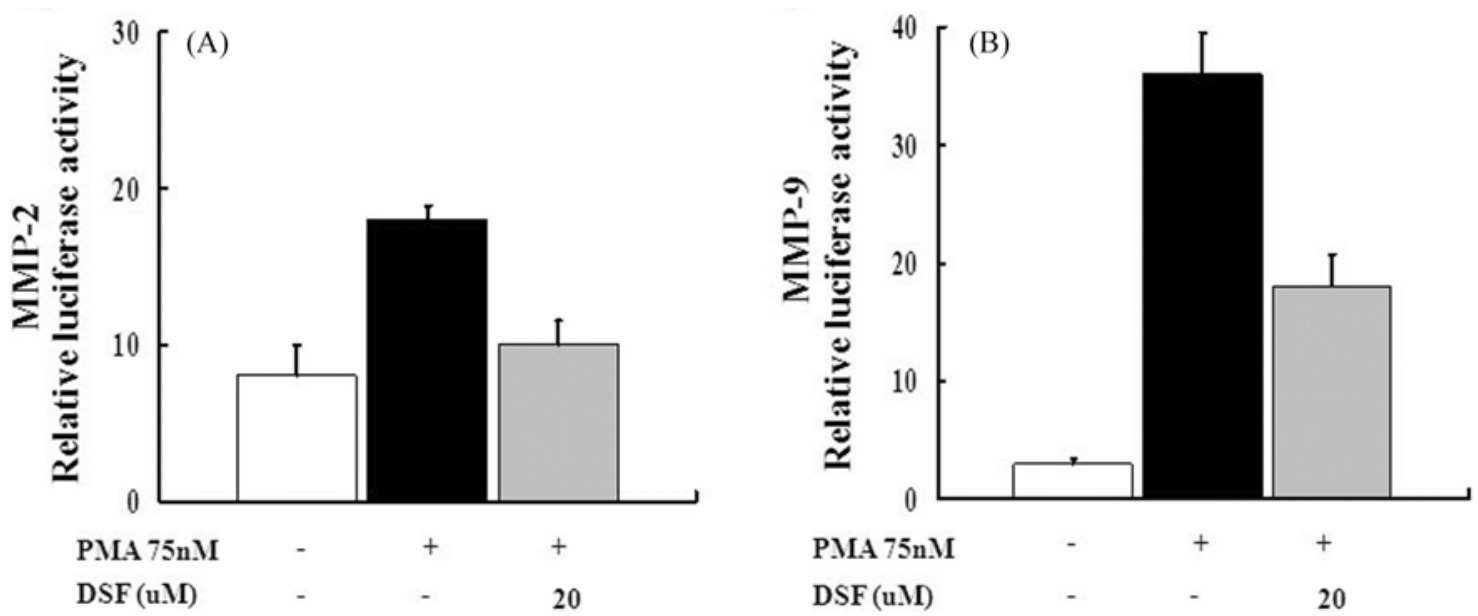

Fig. 5. Effects of disulfiram on the activities that function through MMP-2 (A) and MMP-9 (B) promoter. U2OS cells were transfected with pGL3-MMP-2WT and pGL3-MMP-9WT reporter plasmids, and then cultured with PMA (75 nM) and disulfiram (20 $\mu$ M). U2OS cells transfected with the indicated reporters were cultured with disulfiram $(20 \mu \mathrm{M})$ and/or PMA $(75 \mathrm{nM})$ for $24 \mathrm{~h}$, and luciferase activity in cell extracts was then determined. Values represent the mean of at least three independent experiments. Data represent the mean $\pm \mathrm{S}$.E. of at least three independent experiments. Results were deemed statistically significant using Student's $t$-test.

used clinically in the treatment of alcoholism (Lipsky et al., 2001). Disulfiram has zinc chelating capability and is a zinc ejector (Nash et al., 1998). It has been reported to possess antiretroviral activity and can eject intrinsic zinc out of human immunodeficiency virus (HIV) nucleocapsid protein, induce apoptosis, show metal ion-dependent antineoplastic activity and arrests angiogenesis (McDonnel et al., 1997). Disulfiram has also been shown to directly affect at least three of above traits-inducing apoptosis, acting as an anti-angiogenesis agent and preventing tissue invasiveness and metastasis (Sauna et al., 2005; Chen et al., 2006). However, the mechanism for disulfiram's effects remains unclear, and the use of disulfiram has not yet been reported in the treatment for human osteosarcoma.

In this study, we explored whether disulfiram can modulate cancer invasion and metastasis in cultured human osteosarcoma cells through MMPs by analyzing its effect on MMP-2 and MMP-9. Our results revealed that the non-toxic ranges of disulfiram suppressed MMP-2 and MMP-9 activity and expression, producing an almost complete growth inhibition at $10 \mu \mathrm{M}$ concentration of disulfiram.

Therefore, the inhibitory effect of disulfiram on tumor invasion may be associated with the degradative cascade of the ECM and basement membrane. However, it is not clear whether disulfiram is able to directly inhibit the activity of the active form of MMP. Nonetheless, these results clearly indicate that the anti-invasive effect of disulfiram is associated with inhibition of the enzymatic degradative processes involved in tumor invasion and disulfiram acts as a potent inhibitor of MMP-2 and MMP-9 expression. These results provide evidence suggesting that inhibition of MMPs activity via the chelating agent disulfiram may serve as a therapy to inhibit tumor cell invasion and metastasis. In addition, disulfiram has been shown to directly inhibit the growth of cancer cells both in vitro and in vivo, while divalent metal ions have been shown to enhance its antineoplastic activity (Sauna et al., 2005). Disulfiram inhibited type IV collagen degradation through both the cell-mediated and non-cell-mediated pathways, suggesting that there are at least two possible mechanisms involved in this inhibition process. Our data strongly suggested that disulfiram could directly interact with MMP-2 and MMP-9 proteins through a non-cell-mediated pathway. Furthermore RT-PCR analysis and reporter gene assays revealed that the reduction of the lytic content of MMP-2 and MMP-9 are due to cell-mediated mechanism as well. Disulfiram has the ability to inhibit the invasion of tumor cells by diminishing the efficiency of gene transcription (Fig. 4). Interestingly, our results indicated that TIMP-1 and TIMP-2 mRNA levels were unchanged (Fig. 4C and D). TIMPs play an important role in regulating the activity of the secreted matrix metalloproteases. In addition to their ability to bind at the active site, TIMP-1 and TIMP-2 can form complexes with specific matrix metallproteinases (Stetler-Stevenson et al., 1990). In the present study, the expressions of these inhibitors were not related to the regulation of MMP-2 and MMP-9 expressions. As shown in Fig. 5, disulfiram was found to inhibit luciferase activity in reporter gene assay in a dosedependent manner. These results clearly indicate that the down-regulation of the MMP-2 and MMP-9 genes expression by disulfiram is associated with inhibition of transcription.

MMPs gene expression is primarily regulated at the transcriptional level through AP-1, NF-kB and so forth via mitogen activated protein kinase pathways (Yao et al., 2001). Among the MMPs, the MMP-2 gene expression in cancer cells is dependent on several transcription factors, including Sp1, AP-1, NF-kB and p53 (Ma et al., 2004; Qin et al., 1999). 
It has been previously documented that the MMP-9 promoter has several transcription-factor-binding motifs that can affect

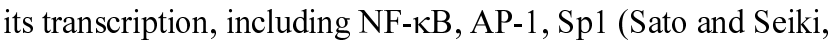
1993; Huang et al., 2005). Disulfiram affected components could downregulate c-Jun/c-Fos and NF- $\mathrm{BB}$ expression levels and then decrease the DNA binding activity of AP-1 and NFКB (Liu et al., 1998; Shian et al., 2003). MMP-2 and MMP-9 may also influence the expression of genes directly responsible for regulating invasion. Taken together, these phenomena may provide possible cell-mediated signaling pathways as a result of disulfiram interference. Disulfiram's suppression of MMP2 and MMP-9 expression could conceivably be through these common factors (AP-1, NF- $\mathrm{kB}$ ). However, its exact mechanism of action still remains elusive. We can therefore theorize that the inhibitory effect of disulfiram on AP-1 and NF-kB activity might explain the resulting suppression of the MMP-2 and MMP-9 genes. In conclusion, we report that disulfiram downregulates the expression of invasion-associated proteins MMP-2 and MMP-9.

These results provide new insights on the potential use of disulfiram in controlling cancer invasion and metastasis. We demonstrated that disulfiram has anti-invasive and antimetastatic activity via the inhibition of MMP-2 and MMP-9 activity in human osteosarcoma cells. Thus disulfiram can be a new anti-cancer drug for the management of human osteosarcoma.

Acknowledgments This work was supported by a grant (Code \#: 20070301-034-001-007-02-00) from BioGreen 21 Program, Rural Development Administration, Republic of Korea.

\section{References}

Brar, S. S., Grigg, C., Wilson, K. S., Holder, W. D., Jr., Dreau, D., Austin, C., Foster, M., Ghio, A. J., Whorton, A. R., Stowell, G. W., Whittall, L. B., Whittle, R. R., White, D. P. and Kennedy, T. P. (2004) Disulfiram inhibits activating transcription factor/cyclic AMP-responsive element binding protein and human melanoma growth in a metal-dependent manner in vitro, in mice and in a patient with metastatic disease. Mol. Cancer Ther. 3, 1049-1060.

Chen. D., Cui, I. C., Yang, H. and Dou, Q. P. (2006) disulfiram, a clinically used anti-alcoholism drug and copper-binding agent, induces apoptotic cell death in breast cancer cultures and zenografts via inhibition of the proteasome activity. Cancer Res. 66, 10425-10433

Cho, J. J., Kang, J. H., Kwak, J. Y., Lee, T. S., Lee, I. S., Park, N. G., Hiroo, N., Jinji, M. and Chang, Y. C. (2007) Ascofuranone suppresses PMA-mediated matrix metalloproteinase-9 gene activation through the Ras/Raf/MEK/ERK-and Ap1-dependent mechanisms. Carcinogenesis 28, 1101-1110.

Christofori, G. (2006) New signals from the invasive front. Nature 441, 444-450.

Dhawan, M., Flora, S. J., Singh, S. and Tandon, S. K. (1989) Chelation of lead during co-exposure to ethanol. Biochem. Int. 19, 1067-1075.
Hong, S., Park, K. K., Magae, J., Ando, K., Lee, T. S., Kwon, T. K., Kwak, J. Y., Kim, C. H. and Chang, Y. C. (2005) Ascochlorin inhibits matrix metalloproteinase- 9 expression by suppressing activator protein-1-mediated gene expression through the ERK1/2 signaling pathway: inhibitory effects of ascochlorin on the invasion of renal carcinoma cells. J. Biol. Chem. 280, 2520225209.

Huang, S. C., Ho, C. T., Lin-Shiau, S. Y. and Lin, J. K. (2005) Carnosol inhibits the invasion of B16/F10 mouse melanoma cells by suppressing metalloproteinase-9 through down-regulating nuclear factor-kappa B and c-Jun. Biochem. Pharmacol. 69, 221232.

Lee, P. P., Hwang, J. J., Murphy, G. and Ip, M. M. (2005) Functional significance of MMP-9 in tumor necrosis factor-induced proliferation and branchig morphogenesis of mammary epithelial cells. Endocrinology 141, 3764-3773.

Li, L., Abe, Y., Kanagawa, K., Shoji, T., Mashino, T., Mochizuki, M., Tanaka, M. and Miyata, N. (2007) Iron-chelating agents never suppress fenton reaction but participate in quenching spin-trapped radicals. Anal. Chim. Acta 599, 315-319.

Liotta, L. A., Steeg, P. S. and Stetler-Stevenson, W. G. (1991) Cancer metastasis and angiogenesis: an imbalance of positive and negative regulation. Cell 64, 327-326.

Lipsky, J. J., Shen, M. L. and Naylor, S. (2001) In vivo inhibition of aldehyde dehydrogenase by disulfiram. Chem. Biol. Interact. 130, 93-102.

Liu, G. Y., Frank, N., Bartsch, H. and Lin, J. K. (1998) Induction of apoptosis by thiuramdisulfides, the reactive metabolites of dithiocarbamates, through coordinative modulation of NFkappaB, c-fos/c-jun, and p53 proteins. Mol. Carcinog. 22, 235-246.

Ma, Z., Chang, M. J., Shah, R., Adamski, J., Zhao, X. and Benveniste, E. N. (2004) Brg-1 is required for maximal transcription of the human matrix metalloproteinase-2 gene. J. Biol. Chem. 279, 46326-46334.

Marikovsky, M., Ziv, V., Nevo, N., Harris-Cerruti, C. and Mahler, O. (2003) $\mathrm{Cu} / \mathrm{Zn}$ superoxide dismutase plays important role in immune response. J. Immunol. 170, 2993-3001.

Masahiro, I., Akira, N., Takashi F., Noriyuki, A., Kengo, M., Toshiaki, E., Koichi, Y., Ryuji, M., Takayuki, Y. and Ryuichi, K. (2002) Anti-invasive effect of MMI-166, a new selective matrix metalloproteinase inhibitor, in Cervical Carcinoma Cell lines. Gynechol. Oncol. 85, 103-107.

Matsunaga, Y., Koda, M. and Murawaki, Y. (2004) Expression of matrix metalloproiteinases (MMPs) and tissue inhibitors of metalloproteinases (TIMPs) in hepatocellular carcinoma tissue, compared with the surrounding non-tumor tissue. Res. Commun. Mol. Pathol. Pharmacol. 115, 143-150.

McDonnel, N. B., De Guznman, R. R., Rice, W. G., Turpin, J. A. and Summers, M. F. (1997) Zine ejection as a new rationale for the use of cystamine and related sidulfide-containing antiviral agents in the treatment of AIDS. J. Med. Chem. 40, 1969-1976.

Nash, T. and Rice, W. G. (1998) Efficacies of zine-finger-active drugs against giardialamblia. Antimicrob. Agents Chemother. 42, 14881492.

Qin, H., Sun, Y. and Benveniste, E. N. (1999) The transcription factors $\mathrm{Sp} 1, \mathrm{Sp} 3$, and AP-2 are required for constitutive matrix metalloproteinase-2 gene expression in astroglioma cells. J. Biol. Chem. 274, 29130-29137.

Sato, H. and Seiki, M. (1993) Regulatory mechanism of $92 \mathrm{kDa}$ type IV collagenase gene expression which is associated with 
invasiveness of tumor cells. Oncogene 8, 395-405.

Sauna, Z. E., Shukla, S. and Ambudkar, S. V. (2005) Disulfiram, an old drug with new potential therapeutic uses for human cancers and fungal infections. Mol. Biosyst. 1, 127-134.

Shen, M. J., Lipsky, J. J. and Naylor, S. (2000) Role of Disulfiram in the In Vitro Inhibition of Rat Liver Mitochondrial Aldehyde Dehydrogenase. Biochem. Pharmacol. 60, 947-953.

Shian, S. G., Kao, Y. R., Wu, F. Y. and Wu, C. W. (2003) Inhibition of invasion and angiogenesis by zinc-chelating agent disulfiram. Mol. Pharmacol. 64, 1076-1084.

Soini, Y., Satta, J., Maatta, M. and Autio-Harmainen, H. (2001) Expression of MMP2, MMP9, MT1-MMP, TIMP1, and TIMP2 mRNA in valvular lesions of the heart. J. Pathol. 194, 225-231.

Soreide, K., Janssen, E. A., Korner, H. and Baak, J. P. (2006) Trypsin in colorectal cancer: molecular biological mechanisms of proliferation, invasion, and metastasis. J. Pathol. 209, 147-156.

Stetler-Stevenson, W. G., Brown, P. D., Onisto, M., Levy, A. T. and Liotta, L.A. (1990) Tissue inhibitor of metalloproteinases-2 (TIMP-2) mRNA expression in tumor cell lines and human tumor tissues. J. Biol. Chem. 265, 13933-13938.

Stewart, D. A., Cooper, C. R. and Sikes, R. A. (2004) Changes in extracellular matrix (ECM) and ECM-associated proteins in the metastatic progression of prostate cancer. Reprod. Biol.
Endocrinol. 2, 2.

Tanimura, S., Kadomoto, R., Tanaka, T., Zhang, Y. J., Kouno, I. and Kohno, M. (2005) Suppression of tumor cell invasiveness by hydrolyzable tannins (plant polyphenols) via the inhibition of matrix metalloproteinase-2/-9 activity. Biochem. Biophys. Res. Commun. 330, 1306-1313.

Woo, J. H., Lim, J. H., Kim, Y. H., Suh, S. I., Min, D. S., Chang, J. S., Lee, Y. H., Park, J. W. and Kwon, T. K. (2004) Resveratrol inhibits phorbol myristate acetate-induced matrix metalloproteinase-9 expression by inhibiting JNK and PKC delta signal transduction. Oncogene 23, 1845-1853.

Yao, J., Xiong, S., Klos, K., Nguyen, N., Grijalva, R., Li, P. and Yu, D. (2001) Multiple signaling pathways involved in activation of matrix metalloproteinase-9 (MMP-9) by heregulin-beta1 in human breast cancer cells. Oncogene 20, 8066-8074.

Yasuhito, K., Ysuyoshi, Y. and Mutsuo, I. (2002) Relationship between expression of matrix metalloproteinase-2 and matrix metalloproteinase- 9 and invasion ability of cervical cancer cells. Oncol. Rep. 9, 565-569.

Yoon, S. O., Kim, M. M. and Chung, A. S. (2001) Inhibitory effect of selenite on invasion of HT1080 tumor cells. J. Biol. Chem. 276, 20085-20092. 